\title{
Phylogeny of Some Mycoplasmas from Ruminants Based on 16S rRNA Sequences and Definition of a New Cluster within the Hominis Group
}

\author{
BERTIL PETTERSSON, ${ }^{1}$ MATHIAS UHLÉN, ${ }^{1}$ AND KARL-ERIK JOHANSSON ${ }^{2,3 *}$ \\ Department of Biochemistry and Biotechnology, The Royal Institute of Technology, S-100 44 Stockholm, ${ }^{1}$ and \\ Department of Bacteriology, National Veterinary Institute, ${ }^{2}$ and Department of Veterinary \\ Microbiology, Swedish University of Agricultural Sciences, ${ }^{3}$ S-750 07 Uppsala, Sweden
}

\begin{abstract}
Almost complete (>96\%) 16S rRNA sequences from nine ruminant mycoplasmas have been determined by solid-phase DNA sequencing. Polymorphisms were found in four of the 16S rRNA sequences, which indicated the existence of two different rRNA operons. Seven polymorphisms were found in Mycoplasma agalactiae, three were found in Mycoplasma bovis, one was found in Mycoplasma alkalescens, and one was found in Mycoplasma bovirhinis. The sequence data were used for construction of phylogenetic trees. All but one of the ruminant mycoplasmas sequenced in this work clustered in the hominis group. A close relationship was found between $M$. agalactiae and $M$. bovis, with a $99 \%$ nucleotide similarity between their $16 \mathrm{~S}$ rRNA sequences. They were also found to be members of the Mycoplasma lipophilum cluster of the hominis group. Furthermore, the 16S rRNA comparisons showed that Mycoplasma alkalescens and Mycoplasma canadense are closely related (>98.5\%), and these species were found to cluster in the Mycoplasma hominis cluster of the hominis group. Interestingly, $M$. bovirhinis grouped in a new phylogenetic cluster of the hominis group. The new cluster, which was supported by bootstrap percentage values, signature nucleotide analysis, and higher-order structural elements, was named the Mycoplasma synoviae cluster. Mycoplasma bovoculi, Mycoplasma conjunctivae, and Mycoplasma ovipneumoniae clustered in the Mycoplasma neurolyticum cluster of the hominis group. Mycoplasma alvi clustered with Mycoplasma pirum in the $M$. pneumoniae cluster of the pneumoniae group.
\end{abstract}

Mycoplasmas (class Mollicutes) are among the smallest microorganisms capable of self-replication and autonomous life. About 150 species are known today, and they are closely related to gram-positive bacteria with a low $\mathrm{G}+\mathrm{C}$ content in the genome (17). Many mycoplasmas are pathogenic for animals, and they are therefore of great concern in veterinary medicine $(18,20)$. Most mycoplasmas are host specific, and ruminants harbor a number of interesting species. Two very important and closely related species are Mycoplasma bovis and Mycoplasma agalactiae. The former causes calf pneumonia, mastitis, and arthritis in cattle $(3,21)$, and the latter species is the causal agent of contagious agalactia in goats and sheep (10). The other species used for $16 \mathrm{~S}$ rRNA sequencing in this study are also of clinical significance, except Mycoplasma alvi. Mycoplasma alkalescens causes arthritis in cattle. $M$. alvi has no known disease-causing potential, but has been isolated from the respiratory tract of cattle. Mycoplasma bovirhinis is often isolated from cattle with respiratory disease, but is probably not a primary pathogen. Mycoplasma bovoculi is associated with conjunctivitis and keratoconjunctivitis in cattle and is often found in the eyes of calves. Mycoplasma canadense is believed to cause mastitis in cattle. Mycoplasma conjunctivae is the causal agent of keratoconjunctivitis in goats and sheep, but it is not clear whether it is a primary or a secondary pathogen. Mycoplasma ovipneumoniae is by far the most common mycoplasma isolated from samples of the respiratory tract of sheep and goats. This organism can cause pneumonia in these animals.

Sequence analysis of $16 \mathrm{~S}$ rRNA has proved to be one of the

\footnotetext{
* Corresponding author. Mailing address: Department of Veterinary Microbiology, Swedish University of Agricultural Sciences, P.O. Box 7073, S-750 07 Uppsala, Sweden. Phone: 46186740 00. Fax: 46 183091 62. Electronic mail address: Kaggen@sva.se.
}

most useful tools for phylogenetic studies (14). A breakthrough in the studies of mycoplasma phylogeny occurred in 1989 when Carl Woese and colleagues published a classic paper on the phylogeny of mycoplasmas based on 16S rRNA sequences generated by direct sequencing of rRNA with reverse transcriptase (24). Proposals for classification of mycoplasmas based on 16S rRNA sequences have been published previously $(12,24)$, and a revised taxonomy of the class $\mathrm{Mol}$ licutes was recently suggested (22). Five phylogenetic groups and several clusters (subgroups) of mycoplasmas were defined (24), and that nomenclature is used in this work. We have determined the 16S rRNA gene sequences of seven additional mycoplasmas from ruminants by solid-phase DNA sequencing and determined their phylogenetic positions. In addition, a sixth novel cluster within the hominis group is described.

\section{MATERIALS AND METHODS}

Bacterial strains, growth conditions, and sample preparation. The mycoplasmas used in this work were obtained from the culture collection of the Mycoplasma Laboratory at the National Veterinary Institute (Table 1). All mycoplasmas were grown in the appropriate medium (2). One milliliter of outgrown suspension cultures was centrifuged, washed once in phosphate-buffered saline, and finally resuspended in $1 \mathrm{ml}$ of water and heated in a boiling water bath for $5 \mathrm{~min}$. The suspensions were then rapidly chilled on ice and stored at $-20^{\circ} \mathrm{C}$ until use.

In vitro amplification of the 16S rRNA gene. Amplification of the $16 \mathrm{~S}$ rRNA gene was performed with a pair of primers complementary to the regions close to the $5^{\prime}$ and the $3^{\prime}$ termini of the gene. A segment consisting of more than $95 \%$ of the gene was thereby amplified. One microliter of the lysed suspensions of each mycoplasma was amplified with the primers RIT593 and RIT620B (Table 2). The PCR products were diluted 50-fold and subjected to two different seminested amplifications. The primers RIT593 and RIT390B were used in one of the amplifications, and RIT388 and RIT620B (Table 2) were used in the second seminested reaction. Biotinylated PCR products, suitable for solid-phase DNA sequencing, were generated with $5 \mathrm{pmol}$ of each primer, and the following thermocycling profile was used. Denaturation at $96^{\circ} \mathrm{C}$ for $15 \mathrm{~s}$ and a combined primer annealing-extension step at $70^{\circ} \mathrm{C}$ for $90 \mathrm{~s}$ were repeated 30 times. A final extension at $72^{\circ} \mathrm{C}$ for $10 \mathrm{~min}$ was also used.

Automated solid-phase DNA sequencing reactions. The biotinylated PCR 
TABLE 1. Mycoplasmas used for solid-phase sequencing of the 16S rRNA genes by the new ALFexpress system

\begin{tabular}{|c|c|c|c|c|c|c|c|}
\hline \multirow{2}{*}{ Species } & \multirow{2}{*}{ Strain } & \multirow{2}{*}{$\begin{array}{c}\text { Major } \\
\text { host }\end{array}$} & \multirow{2}{*}{ Disease } & \multirow{2}{*}{$\begin{array}{c}\text { Glu/Arg } \\
\text { metabolism }\end{array}$} & \multicolumn{2}{|c|}{ Accession no. } & \multirow{2}{*}{$\begin{array}{l}\text { Reference to old } \\
\text { accession no. }\end{array}$} \\
\hline & & & & & Old & New & \\
\hline M. agalactiae & $\mathrm{PG} 2^{\mathrm{T}}$ & Goats & Contagious agalactia & $-1-$ & $\mathrm{M} 24290^{a}$ & U44763 & 24 \\
\hline M. alkalescens & $\mathrm{PG}^{\mathrm{T}}{ }^{\mathrm{T}}$ & Cows & Arthritis & $-1+$ & & U44764 & \\
\hline M. alvi & Isley $^{\mathrm{T}}$ & Cows & & $+1+$ & & U44765 & \\
\hline M. bovirhinis & $\mathrm{PG} 43^{\mathrm{T}}$ & Cows & Mastitis & $+1-$ & $\mathrm{U} 04656^{b}$ & U44766 & 15 \\
\hline M. bovis & Donetta $^{\mathrm{T}}$ & Cows & Respiratory disease & $-1-$ & $\mathrm{U} 02968^{c}$ & $\mathrm{U} 44767^{d}$ & 13 \\
\hline M. bovoculi & $\mathrm{M} 165 / 69^{\mathrm{T}}$ & Cows & Conjunctivitis & $+1-^{e}$ & & U44768 & \\
\hline M. canadense & $275 \mathrm{c}^{\mathrm{T}}$ & Cows & Mastitis & $-1+$ & $\mathrm{U} 04655^{b}$ & $\mathrm{U} 44769$ & 15 \\
\hline M. conjunctivae & $\mathrm{HRC} / 583^{\mathrm{T}}$ & Goats & Keratoconjunctivitis & $+1-$ & & $\mathrm{U} 44770$ & \\
\hline M. ovipneumoniae & $\mathrm{Y}-98^{\mathrm{T}}$ & Sheep & Pneumonia & $+1-$ & $\mathrm{U} 04646^{b}$ & U44771 & 15 \\
\hline
\end{tabular}

${ }^{a}$ Determined by direct RNA sequencing with reverse transcriptase with a few unresolved positions.

${ }^{b}$ About $30 \%$ determined by solid-phase sequencing of a PCR product including the variable regions V6, V7, and V8.

c The complete $16 \mathrm{~S}$ rRNA sequence of the $r m A$ operon was determined after cloning of the gene and conventional DNA sequencing.

${ }^{d}$ Sequence of the $r m B$ operon.

c Some strains are arginine hydrolyzers, but not the type strain.

products were immobilized onto streptavidin-coated super paramagnetic beads (Dynabeads M-280 streptavidin [Dynal AS, Oslo, Norway]), and the strands were separated as described previously $(7,8,15,16,23)$. Both strands were used for sequencing. The sequencing primers were labeled with the indodicarbocyanine dye ALFred (Cy5) phosphoramidite (Pharmacia Biotech, Uppsala, Sweden) for detection with the red laser-based automated laser fluorescent DNA sequencing system ALFexpress (Pharmacia Biotech). The sequences and positions of the sequencing primers are listed in Table 2 . All sequencing primers were purified by reverse-phase liquid chromatography and kindly provided by Pharmacia Biotech. One picomole of the sequencing primer was used in the sequencing reactions.

DNA sequencing with the ALFexpress electrophoresis unit. The gel cassette was assembled with continuous glass spacers with a thickness of $0.3 \mathrm{~mm}$ (thin gel). Fifty milliliters of a gel solution consisting of $5 \%$ Hydro-Link in $1.5 \times$ Tris-borate-EDTA was prepared from 50\% Hydro-Link Long Ranger Gel Solution (AT Biochem, Malvern, Pa.). Polymerization was achieved by addition of $25 \mu \mathrm{l}$ of $N, N, N^{\prime}, N^{\prime}$-tetramethylethylenediamine and $250 \mu \mathrm{l}$ of $10 \%$ ammonium persulfate. The Sanger fragments were separated by electrophoresis at $45^{\circ} \mathrm{C}$ with $0.5 \times$ Tris-borate-EDTA as running buffer. The experimental conditions were $1,500 \mathrm{~V}, 38 \mathrm{~mA}$, and $20 \mathrm{~W}$. The sequence was monitored on-line.

Analysis of 16S rRNA sequences. The $16 \mathrm{~S}$ rRNA sequences from the ruminant mycoplasmas determined in this work were manually aligned with a selection of 16S rRNA sequences from Mycoplasma species, which were retrieved from the data bank of the Ribosomal RNA Database Project (11). Two different alignments were performed. One was composed of representatives of the hominis group, and the other consisted of sequence data from members belonging to the pneumoniae group. A secondary structure model of the 16S rRNA molecule of Mycoplasma hyopneumoniae (6) was used for identification of stem and loop regions in the alignment procedure. The alignments were corrected for ambiguously aligned positions. The phylogenies were inferred by using the neighborjoining program (NEIGHBOR) of Saitou and Nei (19) contained in the Phylogenetic Inference Package, PHYLIP version 3.51c (5). Bootstrap analysis was performed in a set of 500 resamplings with the SEQBOOT program. The similarity matrices were corrected for multiple base changes at single locations by the method of Jukes and Cantor (9).

Nucleotide sequence accession numbers. The sequences of the 16S rRNA genes from the ruminant mycoplasmas have been deposited in GenBank (National Center for Biotechnology Information, Bethesda, Md.) under the accession numbers listed in Table 1.

\section{RESULTS AND DISCUSSION}

Sequences of the 16S rRNA genes. Solid-phase DNA sequencing resulted in nearly full-length sequences $(>96 \%)$ of the 16S rRNA genes of the nine ruminant mycoplasmas listed in Table 1. PCR was used for amplification of the 16S rRNA genes, and sequence data of the termini of the genes are therefore lacking. Polymorphic positions (microheterogeneities) were observed for $M$. alkalescens, $M$. bovirhinis, $M$. bovis, and $M$. agalactiae, and 1, 1, 3, and 7 microheterogeneities were observed in the respective species. All polymorphisms were present in approximately equal amounts (a 1:1 ratio) and indicated the existence of two different 16S rRNA genes in these species. This kind of microheterogeneity has earlier been reported for mycoplasmas of the Mycoplasma mycoides cluster in the spiroplasma group $(15,16)$.

Comparisons with previously published 16S rRNA sequences of Mycoplasma species. A complete sequence from a clone of the 16S rRNA gene was previously published for $M$. bovis Donetta $^{\mathrm{T}}$ (13). That sequence was designated to originate from the $r r n A$ operon. Strain Donetta ${ }^{\mathrm{T}}$ was resequenced in the present work, and the consensus sequences of the $16 \mathrm{~S}$ rRNA genes from both operons were determined. No discrepancies between the sequences were observed, but the genes from the two operons were found to differ by three nucleotides.

TABLE 2. Primers used for PCR and DNA sequencing of mycoplasmas from ruminants

\begin{tabular}{|c|c|c|c|}
\hline Primer $^{a}$ & Position $^{b}$ & Sequence $^{c}$ & Application \\
\hline 593 & $11-35$ & 5'-GTTTGATCCTGGCTCAGGAYDAACG-3' & PCR \\
\hline 388 & $334-356$ & 5'-USP-CCARACTCCTACGGRAGGCAGC-3' & PCR \\
\hline $390 \mathrm{~B}$ & 939-917 & 5'-CTTGTGCGGGYYCCCGTCAATTC-3' & PCR \\
\hline $620 \mathrm{~B}$ & $1542-1520$ & 5'-RSP-GAAAGGAGGTRWTCCAYCCSCAC-3' & PCR \\
\hline USP & & 5'-CGTTGTAAAACGACGGCCAG-3' & Sequencing \\
\hline $583 \mathrm{C}$ & $13-28$ & 5'-TTGATCCTGGCTCAGG-3' & Sequencing \\
\hline $538 \mathrm{C}$ & $800-818$ & $5^{\prime}$-GTAGTCCACGCCGTAAACG-3' & Sequencing \\
\hline $390 \mathrm{C}$ & $939-917$ & 5'-CTTGTGCGGGYYCCCGTCAATTC-3' & Sequencing \\
\hline $597 \mathrm{C}$ & $1175-1193$ & 5'-GAGGAAGGYGRGGATGAYG-3' & Sequencing \\
\hline RSP & & 5'-CACAGGAAACAGCTATGACC-3' & Sequencing \\
\hline
\end{tabular}

${ }^{a} \mathrm{~B}$ and $\mathrm{C}$ indicate that a biotin and an indodicarbocyanine (Cy5), respectively, are coupled to the oligonucleotide. USP and RSP mean that the primer has a handle at the $5^{\prime}$ end with a sequence of the universal sequencing primer.

${ }^{b}$ Numbers are according to the positions in the 16S rRNA gene of Escherichia coli (4).

${ }^{c} \mathrm{D}, \mathrm{R}, \mathrm{S}, \mathrm{W}$, and $\mathrm{Y}$ denote degenerated positions according to the International Union of Biochemistry letter code. 
The sequence of the $\operatorname{rrn} B$ operon could, therefore, easily be deduced from the consensus sequence obtained in this study, by subtraction of the $r m A$ sequence, as described recently (16). A version of the $16 \mathrm{~S}$ rRNA sequence determined by the reverse transcriptase method from $M$. agalactiae was deposited previously (24). The $M$. agalactiae sequence determined in this work was 35 nucleotides shorter in the $3^{\prime}$ end, but was extended by 31 nucleotides in the $5^{\prime}$ end. According to the numbering of the published sequence, a $G$ should be inserted in position 208. Furthermore, all ambiguities were solved, and two positions ( $\mathrm{Y}$ in position 206 and $\mathrm{R}$ in position 1081) were confirmed to be polymorphisms in our study. Five other polymorphic positions were identified, namely, $\mathbf{M}$ (position 161), $\mathrm{K}$ (position 408), W (position 699), Y (position 1065), and $Y$ (position 1152). The fact that two of the seven polymorphisms also were found by direct rRNA sequencing with reverse transcriptase (24) indicates that the $16 \mathrm{~S}$ rRNA genes of both operons of $M$. agalactiae are transcribed. However, it remains to be shown if these small sequence differences between the 16S rRNA molecules have any biological significance. The remaining five polymorphisms could probably not be identified by sequencing with reverse transcriptase and evaluation by autoradiography, which has less resolving power than the ALFexpress system. None of the polymorphisms which occurred in stem regions in the 16S rRNA molecule has resulted in a nucleotide substitution in the complementary position of the stem. This is in agreement with recent findings for the members of the $M$. mycoides cluster (16).

Phylogenetic analysis of mycoplasmas from ruminants. The final alignments consisted of 1239 and 1341 nucleotides of the 16S rRNA sequences from mycoplasmas from ruminants determined in this study and selected Mycoplasma species from the hominis and the pneumoniae groups, respectively. Acholeplasma laidlawii and Mycoplasma capricolum subsp. capripneumoniae served as out-groups in the phylogenetic trees which are shown in Fig. 1 and 2. The corresponding evolutionary distance matrices are presented in Table 3 and Table 4. Eight of the nine ruminant mycoplasmas were found to be phylogenetically related to the hominis group (Fig. 1). M. ovipneumoniae, $M$. bovoculi, and $M$. conjunctivae clustered with the mycoplasmas belonging to the $M$. neurolyticum cluster. A $95.5 \%$ similarity was found between $M$. bovoculi and $M$. conjunctivae, while $M$. ovipneumoniae is closely related to $M$. hyopneumoniae, which is in agreement with previous findings based on partial sequences of the 16S rRNA gene (15). Furthermore, these three species showed the additional nucleotide (a uridine residue) after position 722 , according to the Escherichia coli numbering system (4), which is a higher-order structural element of the Mycoplasma neurolyticum cluster and which is generally not found in the 16S rRNA molecules of other bacterial species. $M$. alkalescens and $M$. canadense were found to be $98.6 \%$ similar to each other (Table 3), and both clustered in the $M$. hominis cluster of the hominis group. $M$. canadense grouped into this cluster also when partial sequences were used for comparison (15). Two very important mycoplasmas in veterinary medicine, the caprine species $M$. agalactiae and the bovine species $M$. bovis, were both resequenced in this work, and their 16S rRNA sequences were found to be very similar $(>99.8 \%)$. M. agalactiae and $M$. bovis were, on the basis of biochemical reactions, originally classified as subspecies of the same species. However, on the basis of serological and DNA-DNA reassociation experiments, they were later reclassified as separate species (1). The $16 \mathrm{~S}$ rRNA sequence data showed that the type strains of $M$. agalactiae and $M$. bovis are closely related, but not identical. Neither do they share any of the polymorphic positions of their $16 \mathrm{~S}$ rRNA

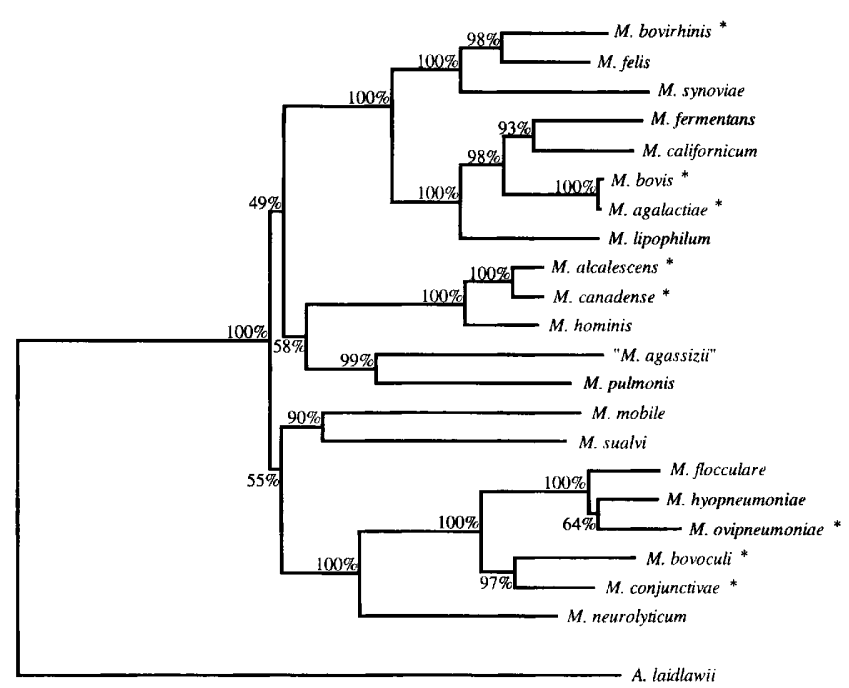

$10 \%$

FIG. 1. Phylogenetic tree based on distance matrix analysis of 1,239 positions in the 16S rRNA gene sequences from 8 mycoplasmas from ruminants sequenced in this work (marked with asterisks) and 13 representatives of the hominis group (24). A. laidlawii was used as the out-group. Bootstrap percentage values obtained from 500 resamplings are given at the nodes. The tree branches into six clusters: the $M$. synoviae cluster, the $M$. lipophilum cluster, the $M$. hominis cluster, the $M$. pulmonis cluster, the $M$. sualvi cluster, and the $M$. neurolyticum cluster (see text for details).

genes. Thus, we believe that there are no reasons to consider another reclassification of these species at this point. An interesting study would be to determine the 16S rRNA sequences of other strains of these species. The dendrogram (Fig. 1) shows that the phylogenetic positions of $M$. agalactiae and $M$. bovis are within the M. lipophilum cluster. The eighth species from ruminants, $M$. bovirhinis, was found to be positioned within a new cluster of the hominis group, which is described in detail below. The ninth mycoplasma from ruminants, $M$. alvi, was $97.8 \%$ similar to Mycoplasma pirum and was positioned within the $M$. pneumoniae cluster of the pneumoniae group of the mollicutes. $M$. alvi also shared the three

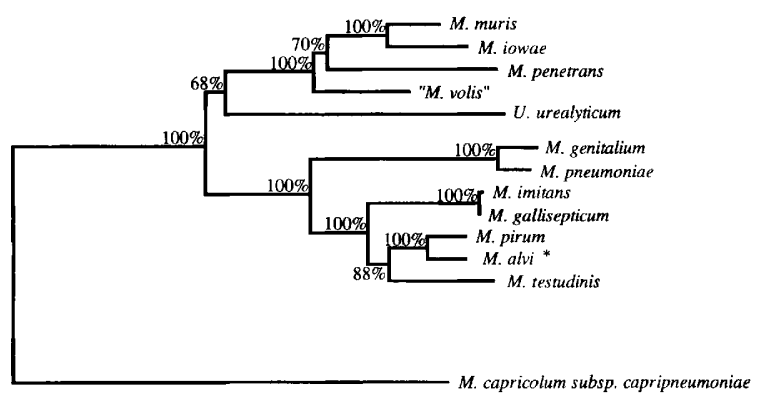

$10 \%$

FIG. 2. Phylogenetic tree based on distance matrix analysis of 1,341 positions in the 16S rRNA gene sequences from $M$. alvi sequenced in this work and 11 representatives of the pneumoniae group (24). The rRNA sequence from the $m A$ operon of $M$. capricolum subsp. capripneumoniae F38 served as the outgroup. Bootstrap percentage values obtained from 500 resamplings are given at the nodes. 
TABLE 3. Similarity matrix of the hominis group ${ }^{a}$

Species

M. synoviae

M. felis
M. bovirhini

92.3

M. bovirhinis $\quad 92.8 \quad 95.7$

M. lipophilum $\quad 89.3 \quad 91.2 \quad 91.2$

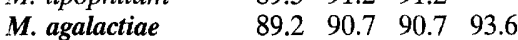

$\begin{array}{llllll}\text { M. bovis } & 89.2 & 90.7 & 90.7 & 93.6 & 99.8\end{array}$

$\begin{array}{lllllll}M \text {. californicum } & 88.0 & 90.3 & 90.7 & 92.5 & 94.6 & 94.4\end{array}$

$\begin{array}{llllllll}M \text {. fermentans } & 88.1 & 89.7 & 89.3 & 93.1 & 95.0 & 94.8 & 95.3\end{array}$

$\begin{array}{llllllllll}M . \text { pulmonis } & 86.1 & 87.6 & 87.8 & 87.8 & 87.3 & 87.2 & 86.4 & 86.4\end{array}$

$\begin{array}{llllllllll}\text { "M. agassizii" } & 84.5 & 85.6 & 84.5 & 85.4 & 84.6 & 84.5 & 84.1 & 84.4 & 90.4\end{array}$

$\begin{array}{lllllllllllll}M . & \text { canadense } & 85.1 & 87.0 & 86.3 & 86.3 & 85.7 & 85.7 & 85.9 & 85.5 & 87.8 & 89.1\end{array}$

$\begin{array}{llllllllllll}M . \text { alkalescens } & 85.7 & 87.2 & 86.2 & 86.5 & 86.0 & 85.8 & 86.2 & 86.1 & 87.6 & 88.4 & 98.6\end{array}$

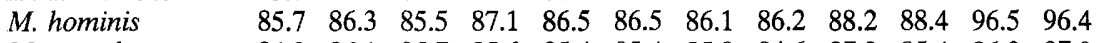

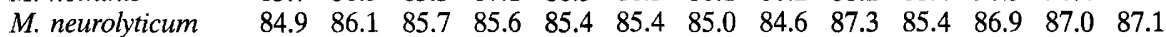

$\begin{array}{llllllllllllllll}M . \text { flocculare } & 83.7 & 85.1 & 83.9 & 83.5 & 83.2 & 83.2 & 82.2 & 82.1 & 84.4 & 83.4 & 84.4 & 84.5 & 84.2 & 88.6\end{array}$

$\begin{array}{lllllllllllllllll}\text { M. ovipneumoniae } & 83.3 & 84.6 & 83.9 & 83.2 & 82.2 & 82.2 & 82.6 & 81.6 & 83.9 & 83.7 & 83.9 & 83.8 & 83.7 & 88.3 & 96.2\end{array}$

$\begin{array}{llllllllllllllllll}M \text {. hyopneumoniae } & 84.3 & 84.9 & 84.3 & 84.4 & 83.2 & 83.2 & 82.5 & 81.9 & 84.7 & 83.7 & 84.0 & 84.1 & 83.5 & 88.7 & 96.9 & 96.8\end{array}$

$\begin{array}{lllllllllllllllllll}\boldsymbol{M} \text {. conjunctivae } & 84.5 & 86.2 & 85.7 & 85.6 & 84.8 & 84.8 & 84.7 & 83.4 & 86.2 & 86.4 & 85.4 & 85.0 & 85.3 & 90.5 & 93.1 & 92.6 & 93.9\end{array}$

$\begin{array}{lllllllllllllllllll}M . \text { bovoculi } & 83.0 & 85.4 & 84.6 & 84.9 & 83.8 & 83.8 & 83.8 & 82.6 & 84.6 & 85.2 & 85.0 & 84.6 & 85.1 & 89.0 & 92.3 & 92.5 & 92.8 & 95.5\end{array}$

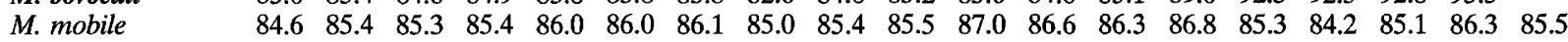

$\begin{array}{lllllllllllllllllllll}\text { M. sualvi } & 84.7 & 87.0 & 85.9 & 85.4 & 86.1 & 86.1 & 84.5 & 84.1 & 85.7 & 84.8 & 87.8 & 88.0 & 87.5 & 87.6 & 84.9 & 83.9 & 84.7 & 85.9 & 84.4 & 88.6\end{array}$

\begin{tabular}{llllllllllllllllllllllll} 
A. laidlawii & 70.5 & 73.1 & 71.7 & 72.6 & 73.5 & 73.3 & 72.1 & 73.0 & 73.6 & 73.3 & 75.3 & 74.6 & 75.6 & 74.9 & 72.2 & 71.9 & 71.8 & 73.2 & 73.6 & 73.3 & 74.1 \\
\hline
\end{tabular}

${ }^{a}$ Species sequenced in this work are in boldface.

synapomorphies with the members of the pneumoniae group (24).

rRNA-based phylogeny in relation to some biochemical reactions. The two most important biochemical reactions used to differentiate mycoplasmas are glucose fermentation $\left(\mathrm{Glu}^{+}\right)$ and arginine hydrolysis $\left(\mathrm{Arg}^{+}\right)$. M. alvi is the only species sequenced in this work which clustered in the pneumoniae group, and it also has a unique biochemical profile $\left(\mathrm{Glu}^{+}\right.$and
$\mathrm{Arg}^{+}$) compared with the other sequenced species. M. pirum, which is phylogenetically close to $M$. alvi, has the same biochemical profile. M. agalactiae and $M$. bovis, which clustered together in the Mycoplasma lipophilum cluster of the hominis group, have a biochemical profile ( $\mathrm{Glu}^{-}$and $\mathrm{Arg}^{-}$) which they share with Mycoplasma californicum in this cluster. M. bovoculi, $M$. conjunctivae, and $M$. ovipneumoniae in the $M$. neurolyticum cluster of the hominis group have another biochemical profile

TABLE 4. Similarity matrix of the pneumoniae group ${ }^{a}$

\begin{tabular}{|c|c|c|c|c|c|c|c|c|c|c|c|c|}
\hline \multirow[b]{2}{*}{ Species } & \multicolumn{12}{|c|}{$\%$ similarity to species: } \\
\hline & 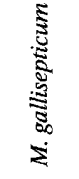 & 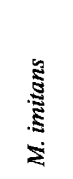 & 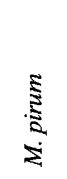 & ปี & 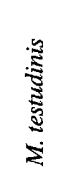 & 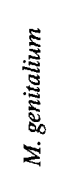 & 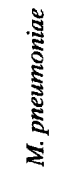 & 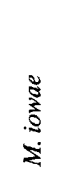 & 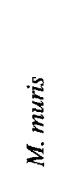 & 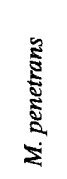 & \begin{tabular}{l}
3 \\
\multirow{3}{3}{} \\
3 \\
3
\end{tabular} & 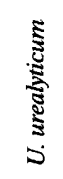 \\
\hline \multicolumn{13}{|l|}{ M. gallisepticum } \\
\hline M. imitans & 99.9 & & & & & & & & & & & \\
\hline M. pirum & 93.8 & 93.7 & & & & & & & & & & \\
\hline M. alvi & 93.8 & 93.7 & 97.8 & & & & & & & & & \\
\hline M. testudinis & 93.4 & 93.5 & 94.8 & 95.1 & & & & & & & & \\
\hline M. genitalium & 88.9 & 88.9 & 89.6 & 88.9 & 88.3 & & & & & & & \\
\hline M. pneumoniae & 88.7 & 88.7 & 90.1 & 89.6 & 88.9 & 98.0 & & & & & & \\
\hline M. iowae & 84.1 & 84.0 & 85.7 & 86.0 & 84.0 & 82.3 & 82.7 & & & & & \\
\hline M. muris & 85.5 & 85.4 & 86.8 & 86.7 & 85.2 & 83.7 & 83.5 & 96.3 & & & & \\
\hline M. penetrans & 84.2 & 84.1 & 85.2 & 85.0 & 82.8 & 82.7 & 82.6 & 91.5 & 91.8 & & & \\
\hline “M. volis" & 86.5 & 86.6 & 87.9 & 88.3 & 86.2 & 83.1 & 83.6 & 93.4 & 93.7 & 92.0 & & \\
\hline U. urealyticum & 83.9 & 83.8 & 85.1 & 84.6 & 82.6 & 81.6 & 81.8 & 85.5 & 86.4 & 84.3 & 86.9 & \\
\hline $\begin{array}{l}\text { M. capricolum subsp. capri- } \\
\text { pneumoniae } \mathrm{F} 38 \mathrm{~mA}\end{array}$ & 74.7 & 74.6 & 74.6 & 75.0 & 73.8 & 73.7 & 73.9 & 75.4 & 75.1 & 73.9 & 75.8 & 74.3 \\
\hline
\end{tabular}

\footnotetext{
${ }^{a}$ The species sequenced in this work is in boldface.
} 
TABLE 5. Unique nucleotide positions in the $16 \mathrm{~S}$ rRNA molecule of the $M$. synoviae cluster

\begin{tabular}{|c|c|c|c|c|c|}
\hline \multirow{2}{*}{$\begin{array}{l}\text { Position } \\
\text { of base } \\
\text { or pair }\end{array}$} & \multicolumn{5}{|c|}{ Residue in: } \\
\hline & $\begin{array}{l}M \text {. synoviae } \\
\text { cluster }^{b}\end{array}$ & $\begin{array}{l}\text { Hominis } \\
\text { group }^{c}\end{array}$ & Mycoplasmas $^{d}$ & Eubacteria $^{e}$ & Exception $(\mathrm{s})^{r}$ \\
\hline $157 \cdot 164$ & $G \cdot U$ & $\mathrm{U} \cdot \mathrm{A}$ & $\mathrm{U} \cdot \mathrm{A}, \mathrm{U} \cdot \mathrm{G}$ & No signature & $\mathrm{G} \cdot \mathrm{U}:$ A. laidlawii, $A$. anaerobium \\
\hline $158 \cdot 163$ & $A \cdot U$ & $\mathrm{G} \cdot \mathrm{C}$ & $\mathrm{C} \cdot \mathrm{G}, \mathrm{G} \cdot \mathrm{C}, \mathrm{U} \cdot \mathrm{A}$ & No signature & A: $A$. anaerobium \\
\hline 610 & A & $\mathrm{U}$ & $\mathrm{G} / \mathrm{U}$ & No signature & \\
\hline 746 & $\mathrm{C}^{\mathrm{g}}$ & $\mathrm{U}$ & $\mathrm{A} / \mathrm{G} / \mathrm{U}$ & $\mathrm{A} / \mathrm{G} / \mathrm{U}$ & $\begin{array}{l}\text { C: Fibrobacter spp. and Plnc (2), Peptostreptococcus subgroup (5), Meth- } \\
\text { ylomonas spp. (2), Porphyromonas spp. (2), Moraxella spp. (4) Methy- } \\
\text { lococcus sp. (1), Staphylococcus spp. (4) }\end{array}$ \\
\hline $824 \cdot 876$ & $\mathrm{G} \cdot \mathrm{C}$ & $\mathrm{C} \cdot \mathrm{G}$ & $\mathrm{U} \cdot \mathrm{A}, \mathrm{C} \cdot \mathrm{G}, \mathrm{A} \cdot \mathrm{U}$ & $\mathrm{U} \cdot \mathrm{A}, \mathrm{C} \cdot \mathrm{G}, \mathrm{A} \cdot \mathrm{U}$ & $\begin{array}{l}\mathrm{G} \cdot \mathrm{C}: \text { A. anaerobium, } \beta-\mathrm{Prpl} \text { (some), Chloroflexus subdivision (some), } \\
\text { FCB (some), Aeromonas group, enterics and relatives, Haemophilus- } \\
\text { Pasteurella group }\end{array}$ \\
\hline
\end{tabular}

\footnotetext{
a Positions are given according to the $E$. coli numbering (4).

${ }^{b}$ Residue present in mycoplasmas of the $M$. synoviae cluster with no exception, if not otherwise stated (i.e., position 746 ).

Dominating residue found in mycoplasmas of the hominis group with no more than one exception.

${ }^{d}$ Dominating residue found in other mycoplasmas.

${ }^{e}$ Dominating residue found in eubacteria in a position with a signature feature for the $M$. synoviae cluster.

$f$ Numbers in parentheses denote the number of sequences in which the actual residue is found. Groups and subgroups are according to those defined by The Ribosomal Database Project (11). Abbreviations: Plnc; Planctomyces spp. and relatives; $\beta$-Prpl, The beta subdivision of purple bacteria (class Proteobacteria); FCB, Flexibacter-Cytophaga-Bacteroides phylum.

${ }^{g}$ Except $M$. gallinaceum, which has a uridine residue in this position.
}

$\left(\mathrm{Glu}^{+}\right.$and $\left.\mathrm{Arg}^{-}\right)$which they also share with $M$. neurolyticum. $M$. bovirhinis, which clustered in the proposed new $M$. synoviae cluster of the hominis group, also has this biochemical profile in common with $M$. synoviae and $M$. felis of this cluster. The biochemical profile of $M$. alkalescens and $M$. canadense is $\left(\mathrm{Glu}^{-}\right.$and $\left.\mathrm{Arg}^{+}\right)$, which they have in common with $M$. hominis. These species belong to the $M$. hominis cluster of the hominis group. In general, closely related species within a phylogenetic cluster have the same biochemical profile, whereas more distantly related species within a cluster can have different biochemical profiles. Thus, glucose fermentation and arginine hydrolysis cannot be used to study the relationships between mollicutes, which has been pointed out earlier (24). However, these phenotypes are useful as a step in the identification of species.

The $M$. synoviae cluster: a new phylogenetic cluster within the hominis group. Five distinct clusters of the hominis group were defined by Woese and coworkers (24). These were denoted as the $M$. hominis cluster, the $M$. lipophilum cluster, the $M$. pulmonis cluster, the $M$. sualvi cluster, and the $M$. neurolyticum cluster, with a nomenclature associated with a representative member of each of the clusters. The phylogenetic tree of the hominis group constructed in this work, represented by Fig. 1, formed six distinct clusters. Therefore, we aligned all currently deposited and almost complete $16 \mathrm{~S}$ rRNA sequences from 35 mycoplasmas belonging to the hominis group (data not shown). The mycoplasma species phylogenetically positioned at the branch of $M$. synoviae were found to have several features in common to justify designation of a new cluster within the hominis group. Despite long branches (Fig. 1) and distant relations (Table 3), we consider "Mycoplasma agassizii", $M$. pulmonis, Mycoplasma mobile, and $M$. sualvi to be two clusters that consist of two species each. These four species can also be seen as four different and phylogenetically true clusters (each consisting of only one species). However, we believe that the latter phylogeny has to be proven when 16S rRNA sequences from close relatives from each of these four species have been generated. Like the species of the neighboring $M$. lipophilum cluster, the members of the $M$. synoviae cluster (including the partially sequenced species $M$. canis and the buzzard isolate Mycoplasma sp. M207/78) have two extra residues inserted in the locale between positions 722 and 723 of the
rRNA molecule. These residues can be regarded as prone to mutation, since they vary between the different species of the two clusters. A discerning oligonucleotide pattern that can be regarded as significant was not found between the two clusters. This attribute occurs nowhere else among the procaryotes and supports the tree (Fig. 1) in that the M. lipophilum cluster and the $M$. synoviae cluster originate from a common and ancestral branch. In contrast, the $M$. synoviae cluster does not share the other two higher-order structural elements considered to be idiosyncratic for the $M$. lipophilum cluster (24). These structural elements comprise the 5-bp truncation of the helix between positions 1435 and 1466 of the $16 \mathrm{~S}$ rRNA molecule and the insertion of a $\mathrm{C} \cdot \mathrm{G}(\mathrm{U} \cdot \mathrm{G}$ for $M$. meleagridis, which belongs to the $M$. lipophilum cluster) base pair in the stalk and a one-nucleotide reduction in the terminating loop at position 420. The latter feature was seen in only 8 of 10 members of the M. lipophilum cluster and is rarely found among the eubacteria. A loop-proximal pair is, however, also found in all members of the pneumoniae group, but with the composition $\mathrm{U} \cdot \mathrm{A}$ in the vicinity of the loop at position 420 . Another higher-order structural feature for the members of the $M$. synoviae cluster was their lack of the helix between positions 1126 and 1144. This truncation is also present in the $16 \mathrm{~S}$ rRNA molecule of the members of the pneumoniae group. As for the pneumoniae group, the helix was found to be replaced by four to five nucleotides, but with a higher variability in the nucleotide composition for the $M$. synoviae cluster. Furthermore, the $M$. synoviae cluster can be characterized by several signature nucleotides and unique nucleotides (Table 5). The $\mathrm{G} \cdot \mathrm{U}$ and A - U base pairs at positions $157 \cdot 164$ and $158 \cdot 163$, respectively, terminated by the loop at 159 constitute the unique oligonucleotide, TGAGAAATTA (unique nucleotides are in boldface), for this cluster compared with other mycoplasmas. However, this oligonucleotide sequence is shared with Asteroleplasma anaerobium. Moreover, the positions 746 (C), 824 $(\mathrm{G})$, and 876 (C) were found to be unique not only among the mollicutes, but also when compared with those of all eubacterial species (Table 5). Furthermore, the branch lengths and bootstrap values in the phylogenetic tree support the introduction of a new cluster in the hominis group, the $M$. synoviae cluster (Fig. 1). The members of the $M$. synoviae cluster are $M$. synoviae, M. felis, Mycoplasma leocaptivus, Mycoplasma gallina- 
ceum, Mycoplasma coragypsum, M. bovirhinis, and Mycoplasma sturni. Partial 16S rRNA sequences indicate that $M$. canis and the buzzard isolate Mycoplasma sp. M207/78 also might be members of the $M$. synoviae cluster, but have to be regarded as tentative members until more information about their $16 \mathrm{~S}$ rRNA sequences has been obtained.

In conclusion, the six clusters in the hominis group which are considered to be true phylogenetic units are (i) the $M$. synoviae cluster ( 7 characterized species), (ii) the $M$. lipophilum cluster (10 characterized species), (iii) the $M$. hominis cluster ( 7 characterized species), (iv) the $M$. pulmonis cluster ( 2 characterized species), (v) the $M$. sualvi cluster ( 2 characterized species), and (vi) the $M$. neurolyticum cluster ( 7 characterized species). However, the branching order among the eight clusters was not considered to be established, except for the $M$. lipophilum cluster and the $M$. synoviae cluster, in that they originated from a common ancestral branch.

\section{ACKNOWLEDGMENTS}

We are grateful to Göran Bölske for providing the mycoplasma strains and for valuable discussions about these organisms. We thank AnnaLena Andersson and Marianne Persson for valuable technical assistance.

This work was financially supported by grants from the Göran Gustafsson Foundation and the Swedish Engineering Science Research Council to M.U. and the Research Foundation of SVA (National Veterinary Institute) to K.-E.J. The project forms a part of the EU research collaboration project COST 826 on "Ruminants' Mycoplasmoses."

\section{REFERENCES}

1. Askaa, G., and H. Erno. 1976. Elevation of Mycoplasma agalactiae subsp. bovis to species rank: Mycoplasma bovis (Hale et al.) comb. nov. Int. J. Syst. Bacteriol. 26:323-325.

2. Bölske, G. 1988. Survey of mycoplasma infections in cell cultures and a comparison of detection methods. Zentralbl. Bakteriol. Hyg. A 269:331-340.

3. Boughton, E. 1979. Mycoplasma bovis mastitis. Vet. Bull. 49:377-387.

4. Brosius, J., J. L. Palmer, J. P. Kennedy, and H. F. Noller. 1978. Complete nucleotide sequence of a 16S ribosomal RNA gene from Escherichia coli. Proc. Natl. Acad. Sci. USA 75:4801-4805.

5. Felsenstein, J. 1993. PHYLIP: phylogeny inference package (version 3.51c). Department of Genetics, University of Washington, Seattle.

6. Gutell, R. R. 1994. Collection of small (16S- and 16S-like) ribosomal RNA structures: 1994. Nucleic Acids Res. 22:3502-3507.

7. Hultman, T., S. Bergh, T. Moks, and M. Uhlén. 1991. Bidirectional solid phase sequencing of in vitro-amplified plasmid DNA. BioTechniques 10:8493.

8. Hultman, T., S. Ståhl, E. Hornes, and M. Uhlén. 1989. Direct solid phase sequencing of genomic and plasmid DNA using magnetic beads as solid support. Nucleic Acids Res. 17:4937-4946.

9. Jukes, T. H., and C. R. Cantor. 1969. Evolution of protein molecules, p. 21-132. In H. N. Munro (ed.), Mammalian protein metabolism, vol. 3. Academic Press, Inc., New York.

10. Lambert, M. 1987. Contagious agalactia of sheep and goats. Rev. Sci. Technol. Off. Int. Epizoot. 6:677-711.

11. Maidak, B. L., N. Larsen, M. J. McCaughey, R. Overbeek, G. J. Olsen, K. Fogel, J. Blandy, and C. R. Woese. 1994. The Ribosomal Database Project. Nucleic Acids Res. 22:3485-3487.

12. Maniloff, J. 1992. Phylogeny of mycoplasmas, p. 549-559. In J. Maniloff, R. N. McElhaney, L. R. Finch, and J. B. Baseman (ed.), Mycoplasmas: molecular biology and pathogenesis. American Society for Microbiology, Washington, D.C.

13. Mattsson, J. G., B. Guss, and K.-E. Johansson. 1994. The phylogeny of Mycoplasma bovis as determined by sequence analysis of the 16S rRNA gene. FEMS Microbiol. Lett. 115:325-328.

14. Olsen, G. J., and C. R. Woese. 1993. Ribosomal RNA: a key to phylogeny. FASEB J. 7:113-123.

15. Pettersson, B., K.-E. Johansson, and M. Uhlén. 1994. Sequence analysis of $16 \mathrm{~S}$ rRNA from mycoplasmas by direct solid-phase DNA sequencing. Appl. Environ. Microbiol. 60:2456-2461.

16. Pettersson, B., T. Leitner, M. Ronaghi, G. Bölske, M. Uhlén, and K.-E. Johansson. 1996. Phylogeny of the Mycoplasma mycoides cluster as determined by sequence analysis of the 16S rRNA genes from the two rRNA operons. J. Bacteriol. 178:4131-4142.

17. Razin, S. 1992. Mycoplasma taxonomy and ecology, p. 3-22. In J. Maniloff, R. N. McElhaney, L. R. Finch, and J. B. Baseman (ed.), Mycoplasmas: molecular biology and pathogenesis. American Society for Microbiology, Washington, D.C.

18. Ross, R. F. 1993. Mycoplasma-animal pathogens, p. 69-109. In I. Kahane and A. Adoni (ed.), Rapid diagnosis of mycoplasmas. Plenum Press, New York.

19. Saitou, N., and M. Nei. 1987. The neighbor-joining method: a new method for reconstructing phylogenetic trees. Mol. Biol. Evol. 4:406-425.

20. Simecka, J. W., J. K. Davis, M. K. Davidsson, S. E. Ross, C. T. K.-H. Städtlander, and G. H. Cassell. 1992. Mycoplasma diseases of animals, p. 391-415. In J. Maniloff, R. N. McElhaney, L. R. Finch, and J. B. Baseman (ed.), Mycoplasmas: molecular biology and pathogenesis. American Society for Microbiology, Washington, D.C.

21. Ter Laak, E., G. H. Wentink, and G. M. Zimmer. 1992. Increased prevalence of Mycoplasma bovis in The Netherlands. Vet. Q. 14:100-104.

22. Tully, J. G., J. M. Bové, F. Laigret, and R. F. Whitcomb. 1993. Revised taxonomy of the class Mollicutes: proposed elevation of a monophyletic cluster of arthropod-associated mollicutes to ordinal rank (Entomoplasmatales ord. nov.), with provision for familial rank to separate species with nonhelical morphology (Entomoplasmataceae fam. nov.) from helical species (Spiroplasmataceae), and emended descriptions of the order Mycoplasmatales, family Mycoplasmataceae. Int. J. Syst. Bacteriol. 43:378-385.

23. Wahlberg, J., A. Holmberg, S. Bergh, T. Hultman, ánd M. Uhlén. 1992. Automated magnetic preparation of DNA templates for solid phase sequencing. Electrophoresis 13:547-551.

24. Weisburg, W. G., J. G. Tully, D. L. Rose, J. P. Petzel, H. Oyaizu, D. Yang, L. Mandelco, J. Sechrest, T. G. Lawrence, J. van Etten, J. Maniloff, and C. R. Woese. 1989. A phylogenetic analysis of the mycoplasmas: basis for their classification. J. Bacteriol. 171:6455-6467. 